\title{
Novel Surgical Treatments for Gastroesophageal Reflux Disease: Systematic Review of Magnetic Sphincter Augmentation and Electric Stimulation Therapy
}

\author{
Michal Stanak ${ }^{\mathrm{a}, \mathrm{c}}$, Judit Erdos ${ }^{\mathrm{a}}$, Katharina Hawlik ${ }^{\mathrm{a}}$, Tudor Birsan ${ }^{\mathrm{b}}$
}

\begin{abstract}
Electric stimulation therapy (EST) and magnetic sphincter augmentation (MSA) represent novel methods for the surgical treatment of gastroesophageal reflux disease (GERD). The aim of this review was to assess the effectiveness and safety of EST and magnetic sphincter augmentation device (MSAD) comapred to laparoscopic fundoplication (LF) and proton pump inhibitor therapy (in case of EST). We performed a systematic literature search without restrictions on publication dates in five electronic databases (MEDLINE, EMBASE, the Cochrane library, PubMed, and Centre for Reviews and Dissemination), complemented by hand search, search in trial registries, and documentation provided by the manufacturers. No study passed inclusion criteria for analyzing EST effectiveness. Concerning safety, lead erosion through the esophagus and trocar perforation of the small bowel occurred in $2.4 \%$ of patients (in one study). Only the registry study fulfilled inclusion criteria for effectiveness analysis of MSAD. The crucial outcome of GERD-health-related quality of life (HRQL) score improved from 20 to 3 points in MSAD patients, and from 23 to 3.5 points in LF patients. However, the LF patients were in a more severe stage of the disease. The results yield indefinite conclusions about the use of both MSAD and EST. Clinical effectiveness and safety of both MSAD and EST are not sufficiently proven and are yet to be supported by high quality evidence from randomized controlled trials.
\end{abstract}

Keywords: GERD; MSAD; EST; Magnetic; Electric; Esophagus; LES; Reflux

\section{Introduction}

Gastroesophageal reflux disease (GERD) is the most common

Manuscript submitted April 9, 2018, accepted April 24, 2018

aLudwig Boltzmann Institute for Health Technology Assessment, Vienna, Austria

${ }^{\mathrm{b} S t}$. John of God Hospital, Vienna, Austria

${ }^{\mathrm{c} C o r r e s p o n d i n g ~ A u t h o r: ~ M i c h a l ~ S t a n a k, ~ L u d w i g ~ B o l t z m a n n ~ I n s t i t u t e ~ f o r ~}$ Health Technology Assessment, Garnisongasse 7/20, 1090 Vienna, Austria. Email: michal.stanak@hta.lbg.ac.at

doi: https://doi.org/10.14740/gr1024w upper gastrointestinal disease in the Western countries affecting around $15 \%$ of the population $[1,2]$, of which $10-20 \%$ are experiencing weekly symptoms [3]. Of the patients, $25-42 \%$ are refractory to a once-daily proton pump inhibitor (PPI) therapy, of which $25 \%$ would respond to an increase in PPI dosing to twice daily. However, $42 \%$ of GERD patients are dissatisfied with their PPI treatment outcomes [4].

Standard surgical treatment for GERD is laparoscopic fundoplication (LF), performed as a total or partial wrap of the fundus [5]. The most common is a loose (floppy) Nissen fundic wrap including a posterior hiatal hernia repair. However, the technical difficulty and the lack of a standardized fundoplication technique have a high impact on patient outcomes [1]. Furthermore, recovery time can be as long as 4 - 6 weeks and some practitioners recommend dietary restrictions to their patients for a variable period of time after surgery [6].

Magnetic sphincter augmentation (MSA) and electric stimulation therapy (EST) represent novel methods for the surgical treatment of GERD. The goal of both interventions is to reinforce the weak lower esophageal sphincter (LES), but the difference lies in the way it is achieved. The magnetic sphincter augmentation device (MSAD) is a ring of magnetic beads made of titanium that is laparoscopically placed around the lower esophagus just above the stomach. The MSAD aims to help the LES resisting opening to gastric pressures, preventing reflux from the stomach entering into the esophagus. Swallowing forces temporarily break the magnetic bond, allowing food and liquid to pass normally into the stomach. The magnetic attraction of the device closes the LES immediately after swallowing, restoring the body's natural barrier to reflux [7].

The EST comprises of a bipolar stimulation lead with two stitch electrodes, an implantable pulse generator, and an external programmer [8]. The pair of electrodes is placed in the anterior part of the lower esophagus and sutured in place using standard laparoscopic techniques [9]. The wires are then connected to a stimulator placed in the subcutaneous pocket in the left upper quadrant of the abdominal wall [9]. The EST delivers mild electrical signals to the LES throughout the day, aiming to restore normal function of the LES, preventing reflux from the stomach entering the esophagus.

As the incidence of GERD is increasing not only in the Western world, novel therapies that claim to eliminate the technical shortcomings of the standard surgical treatment LF are emerging to address the disease for the large proportion of 
GERD patients refractory to standard medical therapy. As health care systems are facing the increasing demand for new therapies, the Austrian Federal Ministry of Health commissioned us to conduct a review on the effectiveness and safety of the two novel surgical interventions. To our knowledge, this is the first systematic review on MSAD and EST that takes into account only prospective evidence. Our aim was to review the literature on MSAD and EST in order to assess their effectiveness and safety compared to the established surgical alternative LF.

\section{Methods}

\section{Search strategy}

We performed a systematic literature search without any restrictions on the publication date in five electronic databases (MEDLINE, EMBASE, the Cochrane library, PubMed, and in the database of the Centre for Reviews and Dissemination), complemented by a hand search (reviewing the reference lists of included studies), and a search in trial registries (Clinicaltrials.gov, WHO-ICTRP, EU Clinical Trials). Full details of the search strategy (including syntaxes, parameters, and results) are available from the corresponding author upon request. As additional source of information, we considered documentation provided by the manufacturers.

\section{Study selection}

In a two-stage review process, two researchers independently screened abstracts and full-texts articles for eligibility based on the a priori defined inclusion criteria. Discrepancies were resolved by consensus or by consulting a third researcher.

Our inclusion criteria are listed as follows. 1) Sample characteristics: adult patients with chronic ( $>6$ months) GERD diagnosed based on $24 \mathrm{pH}$ monitoring, esophagitis grade $\mathrm{C}$ or lower, typical symptoms of GERD (heartburn or regurgitation), and at least partial response to a therapeutic trial of PPIs. In the EST review, additional criteria included: LES end-expiratory pressure of $5-15 \mathrm{~mm} \mathrm{Hg}$ and peristaltic contractions seen in $\geq 50 \%$ of swallows with a contraction amplitude of $\geq 30 \mathrm{~mm}$ $\mathrm{Hg}$ during baseline esophageal manometry. In both reviews, moderate to severe symptom chronic GERD and refractory GERD were considered. 2) Treatment characteristics: a) implantation of the MSAD using laparoscopic surgery. Product name: LINX ${ }^{\circledR}$ Reflux Management System; b) implantation of the EST device using laparoscopic surgery. Product name: EndoStim $^{\circledR}$ LES Stimulator. 3) Publication related: studies comparing MSAD or EST with LF, the standard surgical treatment of GERD, and studies comparing EST with sham treatment or PPI therapy, reporting on at least one of the efficacy and safety outcomes described below in outcomes of interest. For the analysis of effectiveness, randomized controlled trial (RCTs) and non-RCTs were considered for inclusion while for the analysis of safety, prospective case series were included as well. We applied a language restriction and considered only English (and German for the assessment of MSAD) language articles.

\section{Data extraction}

One researcher extracted data and the other checked the data extraction. Data were recorded in a structured manner as follows: sample characteristics, study characteristics, primary outcome measures, and secondary outcome measures.

\section{Outcomes of interest}

The primary outcomes of interest comprise GERD-health-related quality of life (HRQL) score, adverse device effects (dysphagia, excessive bloating, nausea/vomiting, pain/discomfort, and inability to belch or vomit), and serious adverse device effects (device erosion, removal, migration, malfunction, and trocar perforation of the small bowel). Secondary outcomes included the single elements of the GERD-HRQL score, intermediate outcomes like heartburn, excessive bloating, daily regurgitation, extra-esophageal symptoms, and patient satisfaction, in case the study did not report on the GERD-HRQL score. Furthermore, intermediate outcomes like discontinuation of PPIs, deMeester $\mathrm{pH}$ score, re-hospitalization, re-operation, and hospital discharge were included.

\section{Quality assessment}

We assessed the quality and risk of bias of the included studies using the Institute of Health Economics' quality appraisal checklist for case series [10]. All studies that we included in the review were scored independently by two authors. Based on the data extraction tables, the data on each selected outcome category were synthesized across studies according to GRADE methodology [11].

\section{Results}

\section{MSAD}

\section{Available evidence}

In the systematic literature search for MSAD, we found 214 citations through electronic database search, 58 through hand search, and the manufacturer submitted 16 publications of which we identified one new publication. After removing duplicates, we screened 273 articles. There were 54 full-text articles assessed for eligibility, of which six were studies (five prospective case series and one prospective registry study) that met our inclusion criteria and were included in the qualitative analysis (Fig. 1: PRISMA tree MSAD).

\section{Characteristics of included studies}

Our analysis of the clinical effectiveness of the MSAD included 249 patients, of which 202 underwent the MSA procedure. For analysing safety of the MSAD, 356 patients from prospective 


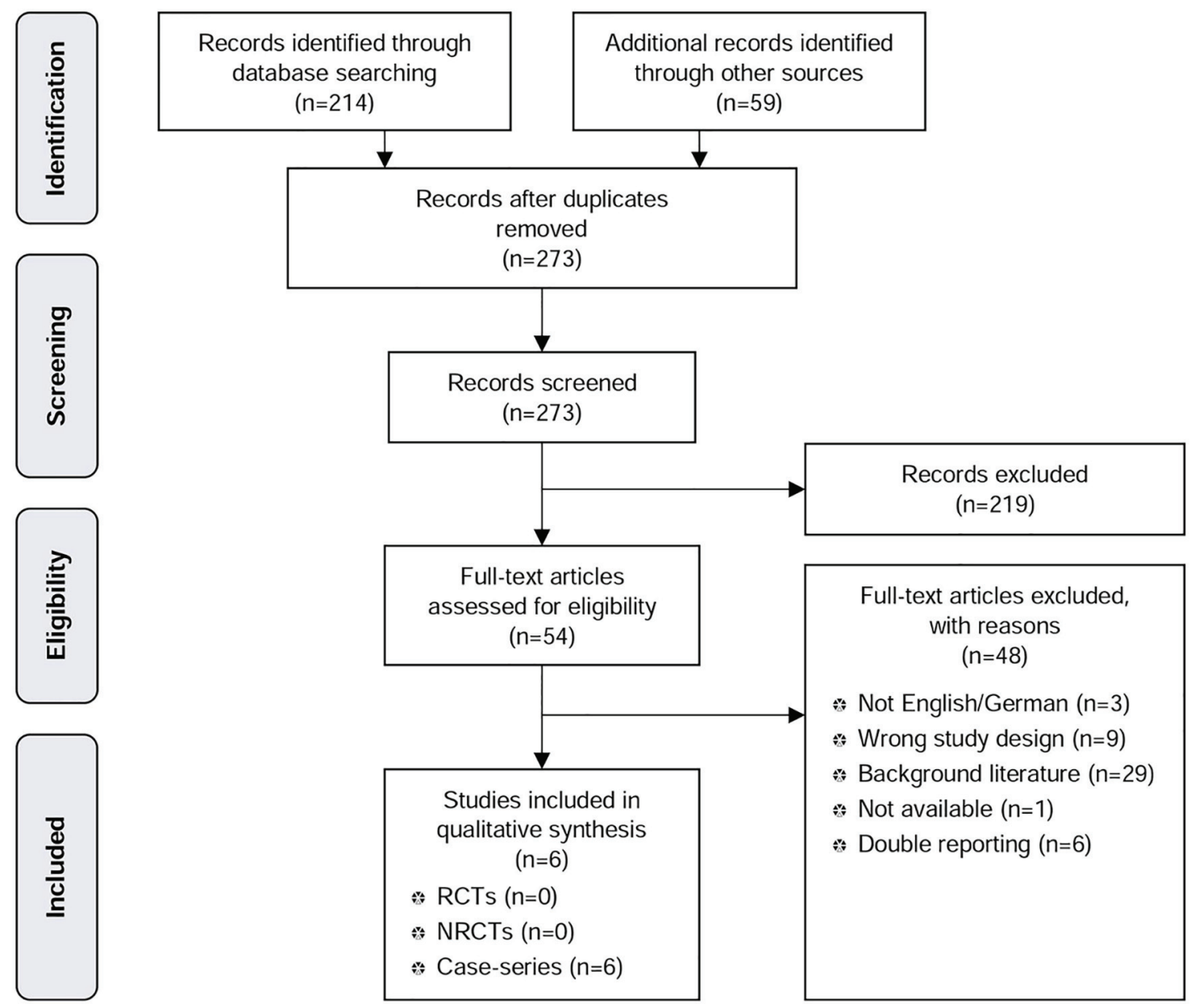

Figure 1. PRISMA tree MSAD.

case series and 202 from the registry study were included. The characteristics of each study are presented in Table 1 [12-21].

\section{Patient characteristics of the included studies}

In the MSAD prospective case series, patient characteristics were homogeneous in terms of Barrett's esophagus, motility disorder, hiatal hernia size, and body mass index (BMI). The inclusion criteria showed some heterogeneity in terms of age, length of GERD symptoms, PPI resistance and responsiveness, and confirmation of reflux by ambulatory esophageal $\mathrm{pH}$ monitoring.

In the controlled registry study, LF patients had a more severe disease in terms of hiatal hernia size, Barrett's esophagus, and esophagitis grade $\mathrm{C}$ and $\mathrm{D}$. The mean BMI score, the number of years on PPIs as well as the number of years with GERD were similar [12]. The baseline characteristics are presented in Table 2 [12-20].

\section{Effectiveness of MSAD}

Only the registry study [12] fulfilled the inclusion criteria for the effectiveness analysis of the MSAD. The GERD-HRQL score improved from 20 to 3 points in MSAD patients, and from 23 to 3.5 points in LF patients. Heartburn improved from the baseline $30.8 \%$ to $3.5 \%$ at 1 -year follow-up in the MSAD group, and from $40 \%$ to $8.5 \%$ in the LF group. Daily regurgitation dropped from $60 \%$ to $13 \%$ in LF patients, whereas in MSAD patients, it dropped from $58.2 \%$ to $3.1 \%$. In terms of frequency of extra-esophageal symptoms, an improvement from $63.9 \%$ to $22.3 \%$ in the MSAD group and from $53.3 \%$ to $17.4 \%$ in the LF group was observed. Of MSAD patients, $81.8 \%$ discontinued PPI therapy compared to $63 \%$ of patients who underwent LF. Dysphagia was not reported, but post-operative excessive bloating appeared in $10 \%$ of MSAD patients, as opposed to $31.9 \%$ of LF patients. Of MSAD patients, $91.8 \%$ were satisfied at follow-up compared to $86.7 \%$ of LF patients.

\section{Safety of MSAD}

Only the registry study [12] allows for the comparison of dysphagia, excessive bloating, inability to belch or vomit, intraoperative complications, re-operation, and re-hospitalization rates between the two groups. Post-operative excessive bloat- 


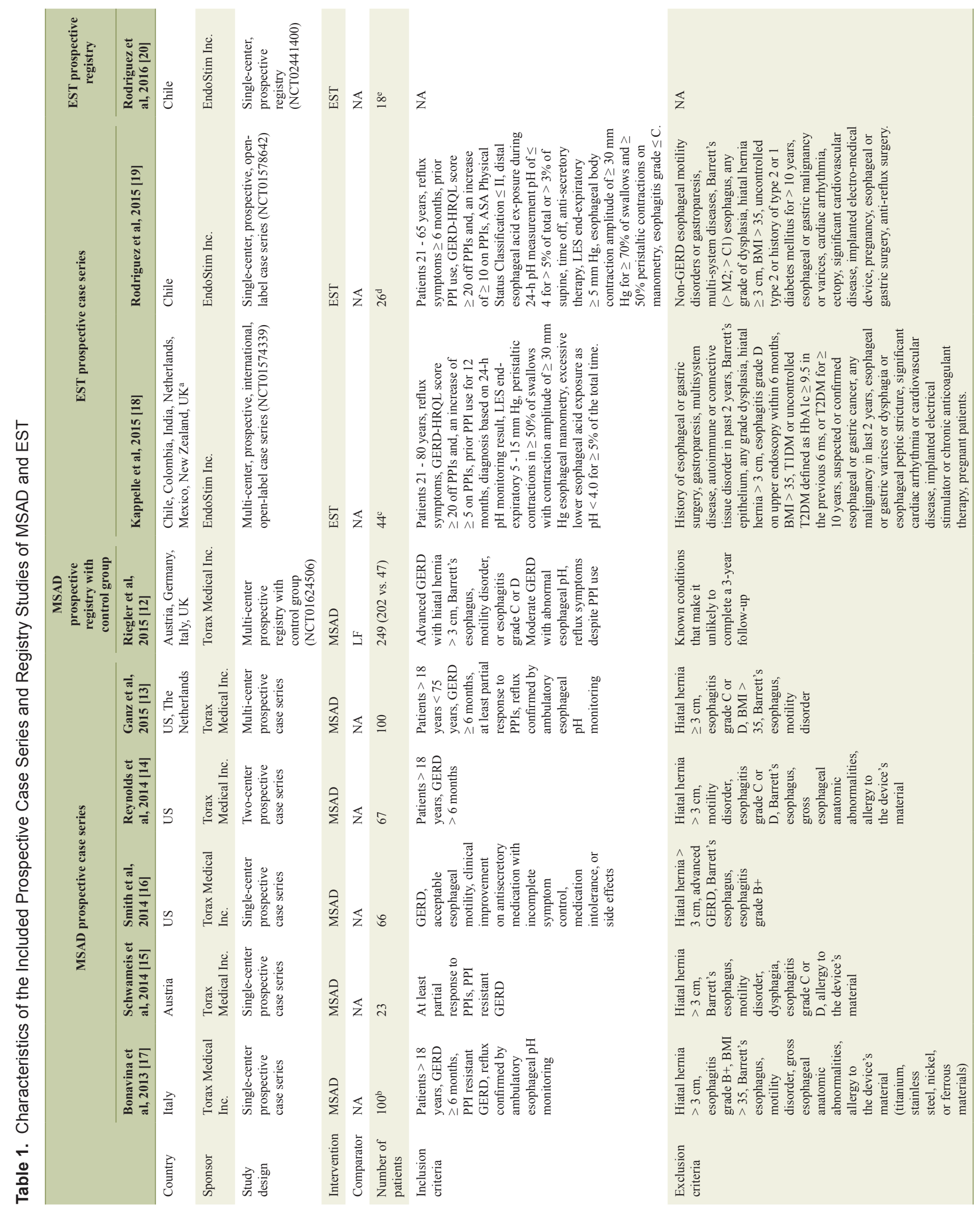




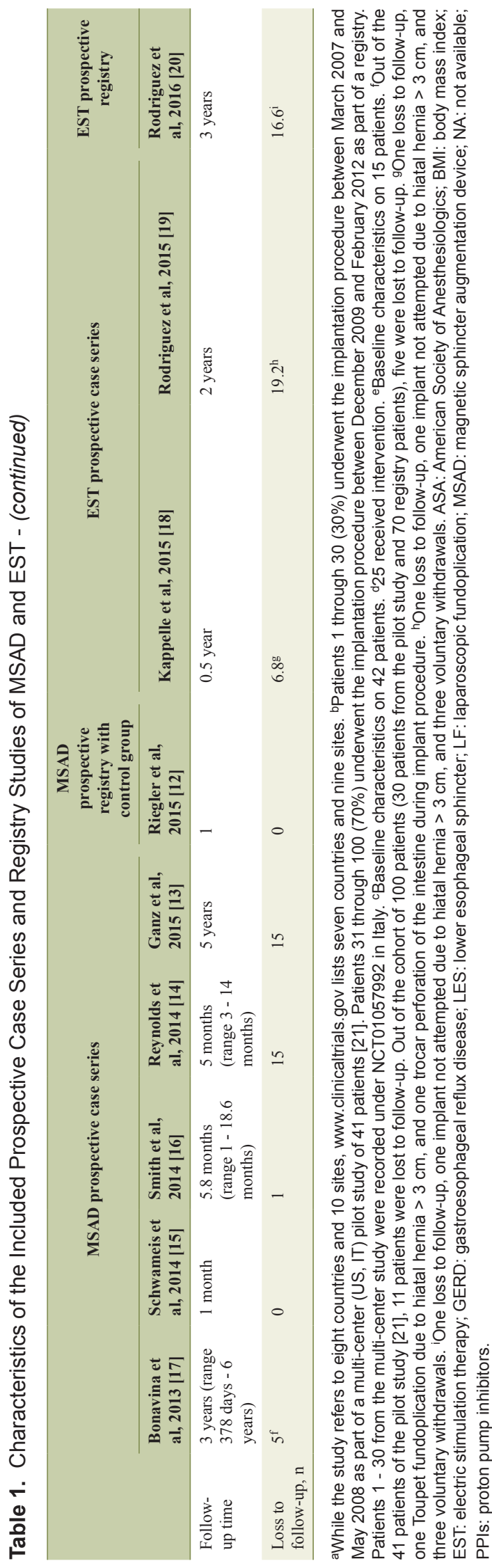

ing was $10 \%$ in MSAD patients, compared to $31.9 \%$ in LF patients. No significant difference was observed between the two groups regarding intraoperative complication rates. Of MSAD patients, $1.6 \%$ experienced the inability to belch compared to $10.1 \%$ of LF patients, $8.7 \%$ experienced the inability to vomit compared to $56.6 \%$ of LF patients, and $7 \%$ of MSAD patients and $10.6 \%$ of LF patients experienced dysphagia at 1-year follow-up. Of MSAD patients, $4 \%$ were re-operated due to device removal compared to $6.4 \%$ in the LF group that were re-operated due to persistent GERD and herniation of the fundic wrap. Hospital re-admission rate was $5.4 \%$ for the MSAD group compared to $4.3 \%$ for the LF group.

Device removal, erosion, malfunction, and migration could be analyzed in both the registry and the case series because the effects directly attributable to the device can be analyzed without a control group, both in case of MSAD as well as EST. MSAD removal was reported in five studies. Two studies with short-term (up to 1 year) follow-up reported no device removals, three studies with long-term (1 - 5 years) followup indicated a device removal rate of $4 \%$ at 1 -year follow-up, $3 \%$ at 3 -year follow-up, and $7 \%$ at 5-year follow-up. Device erosion and migration was reported in three and two studies, respectively, but occurred in none. Device malfunctioning did not occur in the only study that recorded data on this outcome [13] (Table 3 [12-20]).

\section{Evaluation of quality and strength of evidence}

Overall, the quality of evidence for analyzing MSAD was very low. The reasons for downgrading were methodological limitations, inconsistency, and other modifying factors [14-16] (Table 4 [12-20]).

The overall risk of bias was high due to the unclear selection process $[13,16]$, non-consecutive recruitment of study participants $[13,17]$, multiple-reporting $[12,17]$, high loss to follow-up $[13,14]$, and entering the study at a similar point in the disease was either unclear $[12,14,16]$ or clearly not fulfilled $[13,15,17]$ (Table 5 [12-20]). All six studies were sponsored by the manufacturer Torax Medical Inc., and all of the effectiveness outcomes were patient reported, hence subject to a high risk of bias. The inclusion criteria in the registry study were different for the MSAD and LF patients, which undermines the validity of the studies.

\section{EST}

\section{Available evidence}

In the systematic literature search for EST, we found 365 citations through electronic database search, 22 additional citations were found through hand search, and the manufacturer submitted 28 publications, of which we identified 0 new publications. After removing duplicates, we screened 367 articles. Sixty-five full-text articles were assessed for eligibility, of which three were studies (two prospective case series and one prospective registry study) that met our inclusion criteria and 


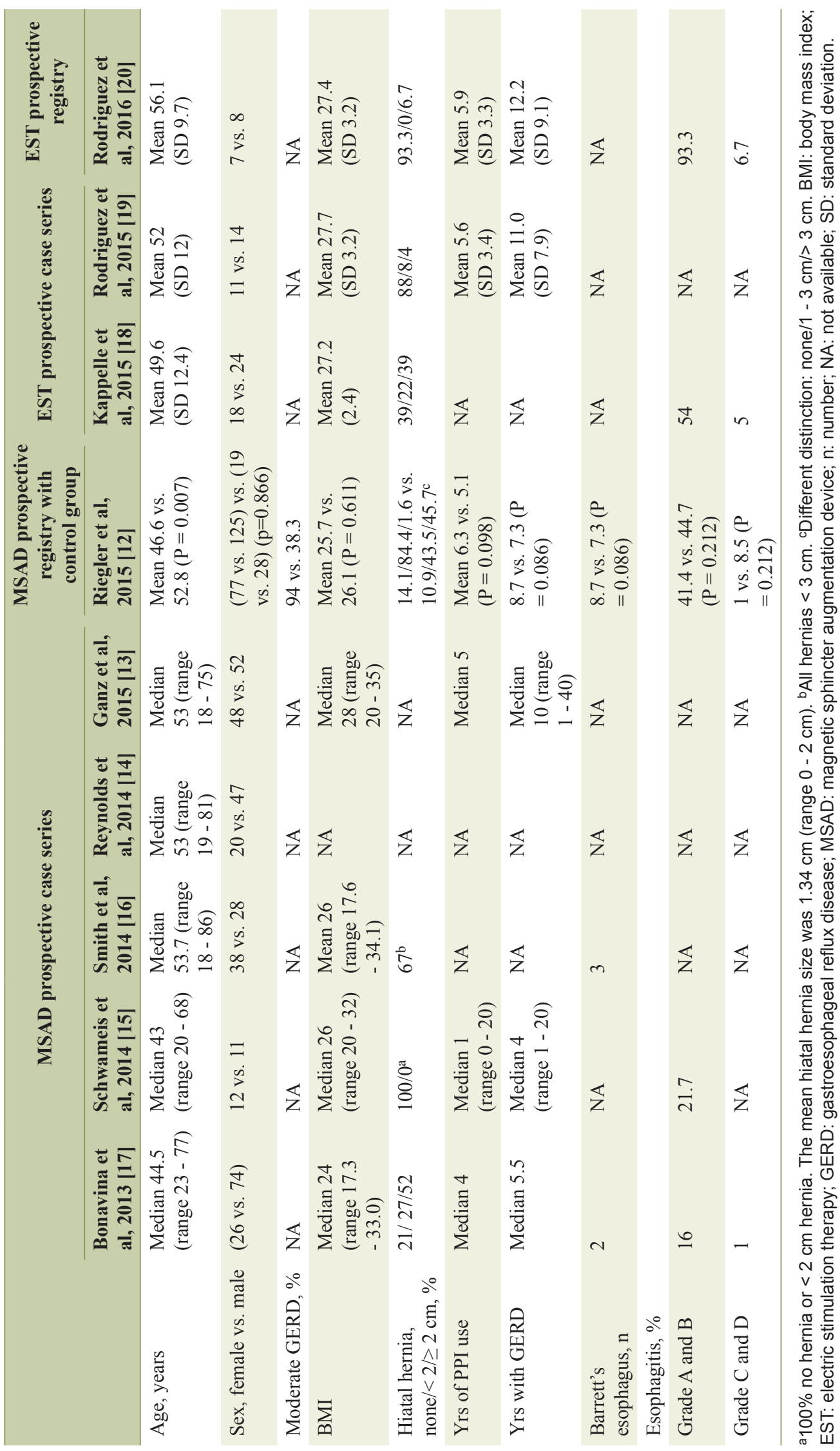




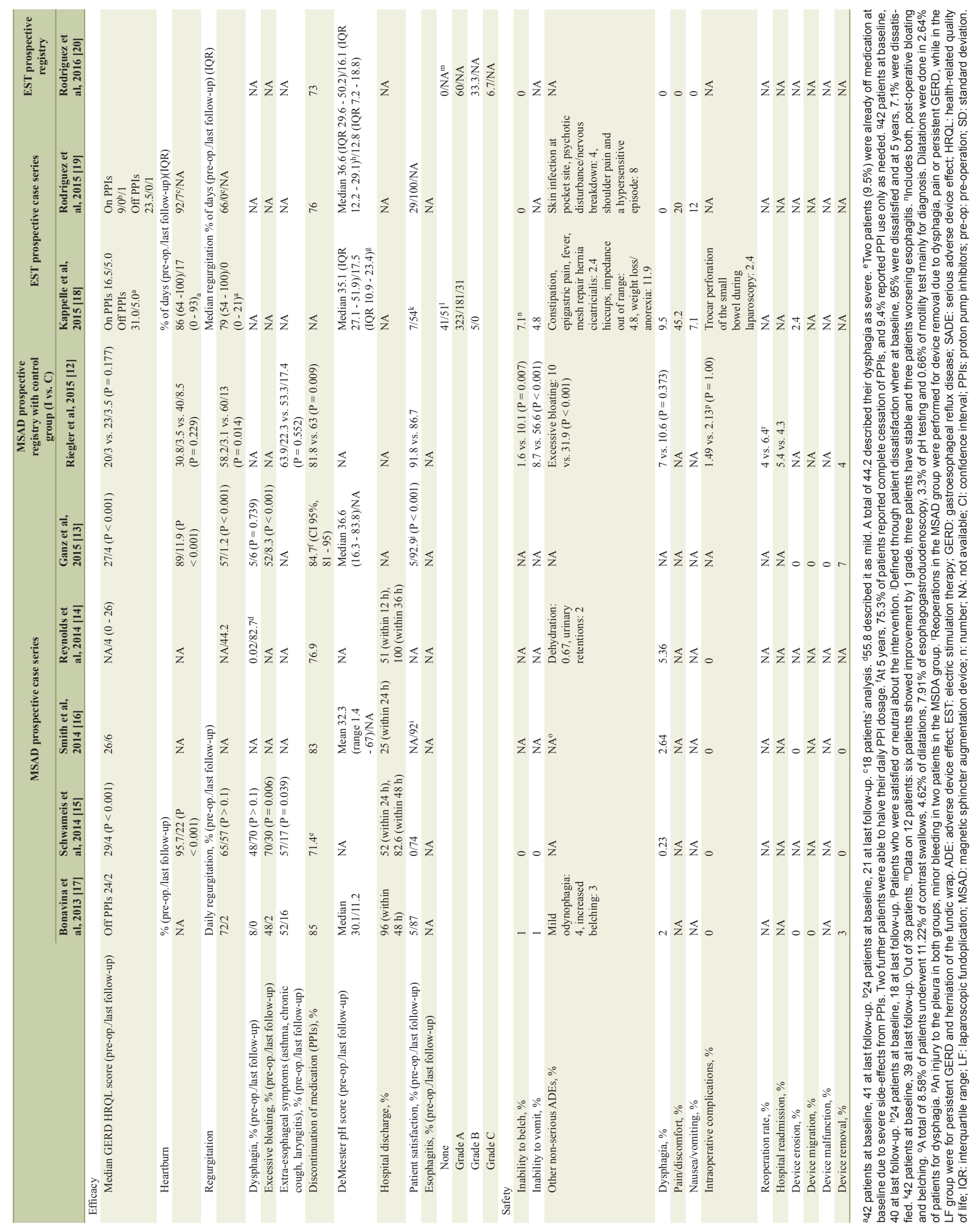


were thus used for the qualitative analysis (Fig. 2: PRISMA tree EST).

\section{Characteristics of included studies}

No study passed the inclusion criteria for analyzing the EST effectiveness. For analyzing safety of the EST, a total of 70 patients were included. The characteristics of each study are presented in Table 1 [12-21].

\section{Patient characteristics of the included studies}

In the prospective case series and the registry study, patient characteristics showed homogeneity in terms of mean age and mean BMI, and heterogeneity in terms of hiatal hernia size. The baseline characteristics are presented in Table 2 [12-20].

\section{Effectiveness of EST}

No study fulfilled the study inclusion criteria for assessing clinical effectiveness of the EST. RCTs and non-RCTs were considered for inclusion, but could not be identified through the systematic literature search.

\section{Safety of EST}

Device related complications were reported in one study with 6 months follow-up [18]. Lead erosion through the esophagus occurred in $2.4 \%$ of patients and was followed by the device explantation. One procedure related complication, trocar perforation of the small bowel during laparoscopy, occurred also in $2.4 \%$ of patients. No other device related complications were reported. Table 3 shows the efficacy and safety outcomes [12-20].

\section{Evaluation of quality and strength of evidence}

The quality of evidence for analyzing EST was very low. This is due to the observational study design, heterogeneity of data, no P-values on pre-post comparison reported, and small sample size (Table 4) [12-20]. Furthermore, the patient group is not representative of the range of GERD patients requiring anti-reflux surgery due to the highly specific patient selection criteria [22].

The overall risk of bias was considered moderate because it was unclear if patients entered the study at a similar point in the disease, if patients were recruited consecutively, and conclusions concerning effectiveness were not supported by the results (Table 5) [12-20]. Internal validity of the trials conducted was undermined by the use of the concomitant therapy of PPIs in all trials. Occasional or regular use of PPIs was reported to be $12 \%$ and $24 \%$ in the case series [18, 19], and $27 \%$ in the registry study [20].

\section{Discussion}

The results of these systematic reviews yield indefinite conclusions about the use of both MSAD as well as EST for moderate to severe GERD patients in whom non-surgical treatment has failed due to incomplete symptom control despite maximum medication treatment or due to severe complications associated with PPI therapy. Both interventions are also an option for patients who opt for surgery despite successful medical management as the use of medications is life-long and non-curative, and for patients with GERD complications such as extraesophageal symptoms (asthma, chronic cough, and laryngitis). Major advantages of MSAD and EST are that they are less invasive and reversible, their implantation is associated with a short learning curve for the surgeon, and they require a shorter hospital stay compared to LF. However, clinical effectiveness and safety of both MSAD and EST are not sufficiently proven by current clinical studies and so are yet to be supported by high quality evidence from RCTs.

\section{Effectiveness and safety}

Currently, there are no comparative data on the EST and only a single controlled registry trial on the MSAD [12]. However, within the registry trial, the differences in inclusion criteria between MSAD and LF patients are such that the comparative value of the study remains undermined (Table 2) [12-20]. Moreover, the fact that LF achieved an improvement in HRQL similar to MSAD despite the fact that patients in the LF group had a more severe disease possibly suggests the LF supremacy. Hence, our systematic review on MSAD [23] draws a different conclusion from systematic reviews and meta-analyses of Skubleny et al [24] as well as Chen et al [25]. Excluding the retrospective trials on methodological grounds [26] and recognizing the vast differences in patient inclusion criteria of the only remaining comparative study [12], we conclude that the effectiveness of MSAD is yet to be established. Also, data on long-term safety considerations such as durability of the devices and device removals need to be considered as well.

\section{Mechanisms behind GERD}

The mechanisms behind GERD need also to be analyzed because they have an impact on the effectiveness of interventions. Only the correct determination of GERD's pathophysiology will help in evaluating the efficacy of anti-reflux treatments. For instance, the LES residual pressure represents a surrogate outcome that the EST claims to improve, yet, the improvement was either reported not to be statistically significant $(P=0.8018)[18]$, or it was not reported at all in the remaining studies $[19,27]$. Other mechanisms possibly having an influence on GERD may have an effect on transient LES relaxation, LES compliance, or the acid pocket [19]. Furthermore, as hiatal hernias make acid reflux more likely [28], it is possible that repairing of the hiatal hernia has an impact on the effectiveness of interventions and hence on GERD symp- 
Table 4. Evidence Profile: Efficacy and Safety of MSAD and EST in GERD Patients

\begin{tabular}{|c|c|c|c|c|c|c|c|}
\hline $\begin{array}{l}\text { No. of studies/ } \\
\text { patients }\end{array}$ & Study design & $\begin{array}{l}\text { Estimate } \\
\text { of effect }\end{array}$ & $\begin{array}{l}\text { Study } \\
\text { limitations }\end{array}$ & $\begin{array}{l}\text { Inconsist- } \\
\text { ency }\end{array}$ & Indirectness & $\begin{array}{l}\text { Other modi- } \\
\text { fying factors }\end{array}$ & $\begin{array}{l}\text { Strength of } \\
\text { evidence }\end{array}$ \\
\hline \multicolumn{8}{|c|}{ Efficacy of MSAD } \\
\hline \multicolumn{8}{|c|}{ Median GERD-HRQL score (pre-op./last follow-up) I vs. C } \\
\hline $1 / 249[12]$ & Prospective registry with control group & $\begin{array}{l}20 / 3 \text { vs. } 23 / 3.5 \\
(P=0.177)\end{array}$ & 0 & NA & 0 & 0 & Moderate \\
\hline \multicolumn{8}{|l|}{ Efficacy of EST } \\
\hline \multicolumn{8}{|c|}{ Safety of MSAD } \\
\hline \multicolumn{8}{|c|}{ Overall complication rate, $\%$ I vs. C } \\
\hline $1 / 249[12]$ & Prospective registry with control group & NA & NA & NA & NA & NA & NA \\
\hline \multicolumn{8}{|c|}{ Intraoperative complications, \% I vs. C } \\
\hline \multicolumn{8}{|c|}{ Device removal, \% } \\
\hline $5 / 336[13-17]$ & $\begin{array}{l}\text { Prospective case series }+ \text { prospective } \\
\text { registry with control group }\end{array}$ & $0-7$ & -1 & -1 & 0 & -1 & Very low \\
\hline \multicolumn{8}{|l|}{ Safety of EST } \\
\hline \multicolumn{8}{|c|}{ Adverse events (AEs) } \\
\hline \multicolumn{8}{|c|}{ Post-operative bloating/belching } \\
\hline $3 / 67[18-20]$ & $\begin{array}{l}\text { Prospective case series }+ \\
\text { prospective registry }\end{array}$ & Not reported & $-1^{\mathrm{a}}$ & $-1^{\mathrm{b}}$ & 0 & $-1^{\mathrm{c}}$ & Very low \\
\hline \multicolumn{8}{|c|}{ Post-operative dysphagia } \\
\hline $3 / 67[18-20]$ & $\begin{array}{l}\text { Prospective case series }+ \\
\text { prospective registry }\end{array}$ & Not reported & -1 & -1 & 0 & -1 & Very low \\
\hline \multicolumn{8}{|c|}{ Serious adverse events (SAEs) } \\
\hline \multicolumn{8}{|c|}{ Trocar perforation of the small bowel } \\
\hline $1 / 42[18]$ & Prospective case series & Not reported & -1 & NA & 0 & -1 & Very low \\
\hline \multicolumn{8}{|c|}{ Device erosion lead through esophagus } \\
\hline $1 / 42[18]$ & Prospective case series & Not reported & -1 & $\mathrm{NA}$ & 0 & -1 & Very low \\
\hline
\end{tabular}

aUnclear risk of bias due to unclear allocation concealment, no blinding, no control group. bHeterogeneous results, no P-value reported. 'small sample size. C: control; EST: electric stimulation therapy; GERD: gastroesophageal reflux disease; I: intervention; LF: laparoscopic fundoplication; MSAD: magnetic sphincter augmentation device; NA: not available; SEA: serious adverse event.

toms.

\section{Ambiguity of alternatives}

Proving the effectiveness of MSAD and EST seems to pose a challenge due to the ambiguity of comparators. On the one hand, LF is presented as a comparator, yet on the other hand, MSAD and EST claim to fill the "therapeutic gap between patients who are dissatisfied with PPI treatment and those who are reluctant to undergo Nissen fundoplication" [29]. In contrast to the LF, the target population of MSAD and EST seems to be represented by patients with a less severe disease. The exact patient group that would benefit most from the treat- 


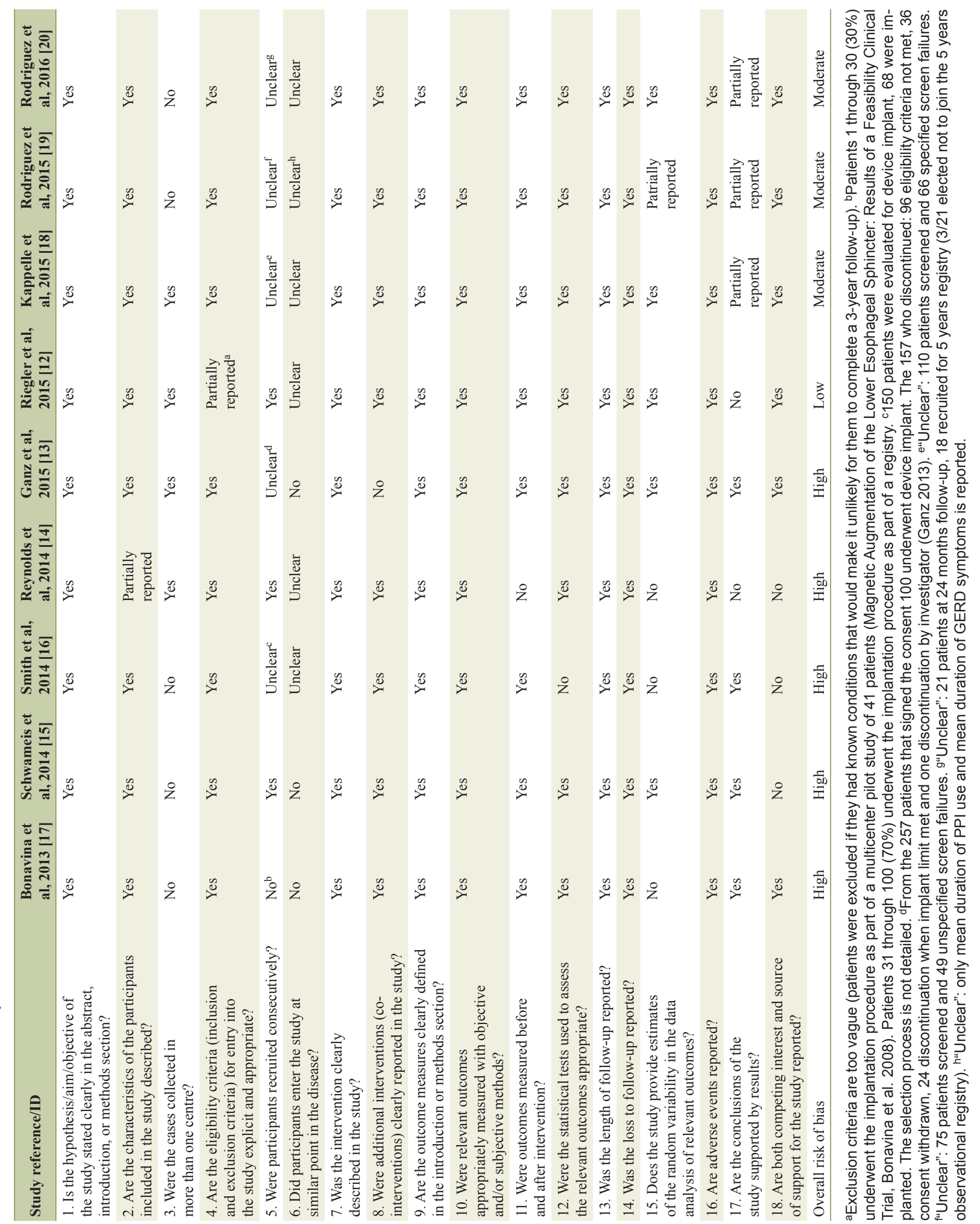




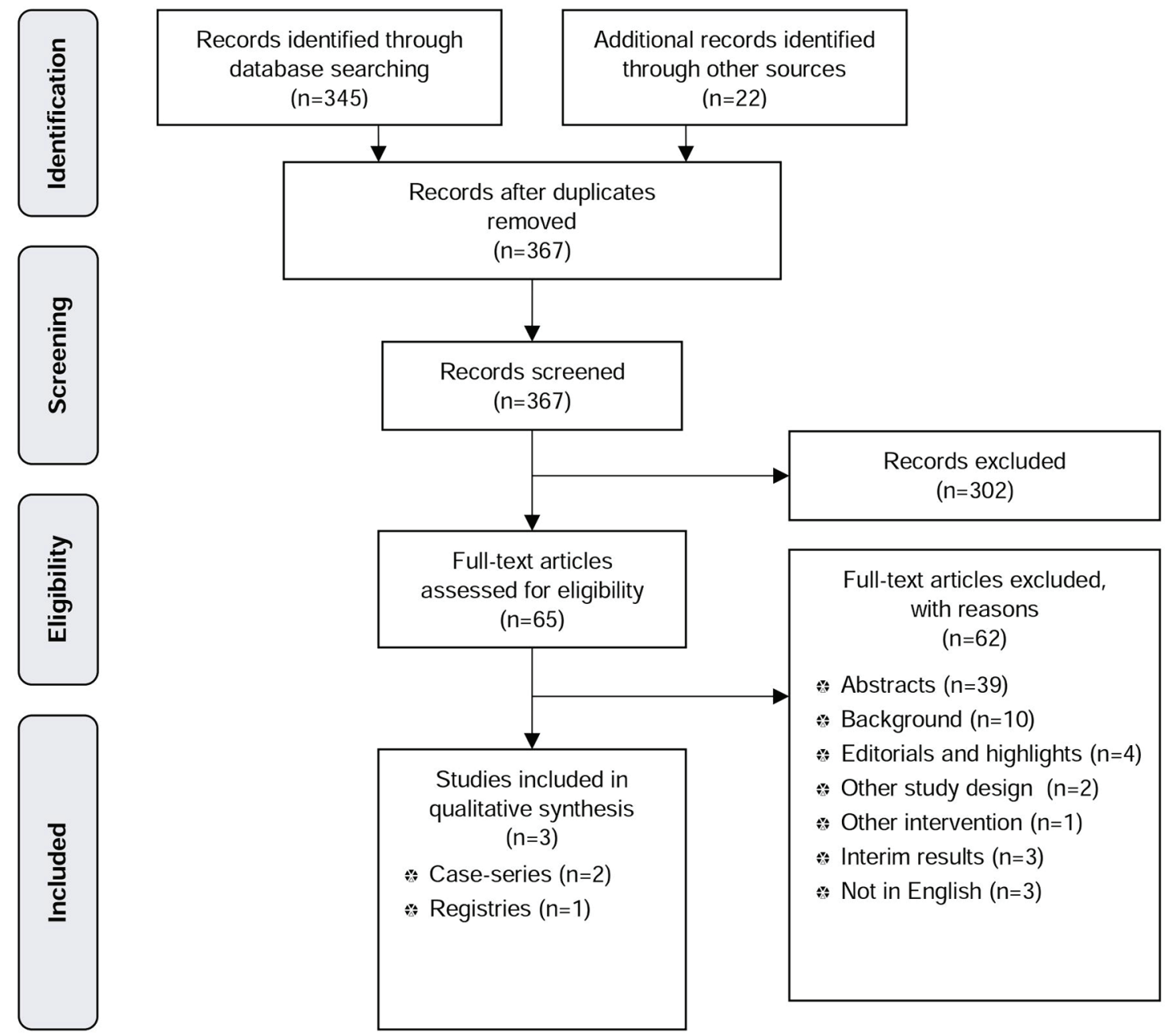

Figure 2. PRISMA tree EST.

ments thus needs to be more clearly defined. Hence, if there really is a therapeutic gap, it is unclear whether it is ethical to conduct an RCT between EST or MSAD, and LF as a comparator at all. Under these assumptions, a sham RCT would be needed to confirm the efficacy of EST. This could be done by implanting the EST device in both groups of patients at the same time, while activating the device straightaway in one group and postponing the activation in the control group. In case of MSAD, an RCT comparing MSAD with PPIs (in less severe cases) or MSAD with LF (in more severe cases) is still needed.

\section{Limitations}

The main limitation to be considered when interpreting these findings is that the MSAD literature search was done in 2016 and was not systematically updated since. However, the search was updated by hand search via searching the web and the clinical trial registries, which did not identify new prospective trials.

\section{Conclusions}

We conclude that the current evidence is not sufficient to prove that the two novel GERD treatments, EST and MSAD, are at least equally effective and as safe as the surgical comparator LF. The quality of the prospective evidence was low as there are only limited comparative data on the effectiveness of MSAD and no comparative data on EST. Both procedures have a relatively good safety-profile, making them a possible treatment option for moderate to severe GERD patients who either refuse further medical therapy or in whom non-surgical treatment has failed. The introduction of the two procedures into clinical practise outside controlled trials seems, however, premature. Controlled trials are essential to confirm safety benefits and establish effectiveness of these new interventions.

\section{Disclosure}

The Ludwig Boltzmann Institute for Health Technology As- 
sessment conducted this study on behalf the Federal Ministry of Health, Austria.

\section{Conflict of Interest}

All authors declare that they have no conflict of interest.

\section{References}

1. SAGES/Society of American Gastrointestinal and Endoscopic Surgeons. Guidelines for surgical treatment of gastroesophageal reflux disease (GERD). 2008.

2. Koop H, Fuchs KH, Labenz J, Jansen L, Messmann H, Miehlke S, Schepp T, Wenzl T. Gastroosophageale Refluxkrankheit unter Federfuhrung Deutschen Gesellschaft fur Gastroenterologie, Verdauungs-und Stoffwechselkrankheiten (DGVS). 2014.

3. Liakakos T, Karamanolis G, Patapis P, Misiakos EP. Gastroesophageal reflux disease: medical or surgical treatment? Gastroenterol Res Pract. 2009;2009:371580.

4. Liu JJ, Saltzman JR. Refractory gastro-oesophageal reflux disease: diagnosis and management. Drugs. 2009;69(14):1935-1944.

5. Zhang H, Dong D, Liu Z, He S, Hu L, Lv Y. Revaluation of the efficacy of magnetic sphincter augmentation for treating gastroesophageal reflux disease. Surg Endosc. 2016;30(9):3684-3690.

6. Singhal V, Khaitan L. Gastroesophageal reflux disease: diagnosis and patient selection. Indian J Surg. 2014;76(6):453-460.

7. Torax Medical. The LINX Reflux Management System: Stop reflux at its source. 2016.

8. Rodriguez L, Rodriguez P, Gomez B, Ayala JC, Saba J, Perez-Castilla A, Galvao Neto M, et al. Electrical stimulation therapy of the lower esophageal sphincter is successful in treating GERD: final results of open-label prospective trial. Surg Endosc. 2013;27(4):1083-1092.

9. Bonavina L, Attwood S. Laparoscopic alternatives to fundoplication for gastroesophageal reflux: the role of magnetic augmentation and electrical stimulation of the lower esophageal sphincter. Dis Esophagus. 2016;29(8):9961001.

10. Moga C, Guo B, Schopflocher D, Harstall C. Development of a quality appraisal tool for case series studies using a modified Delphi Technique Institute of Health Economics, Edmonton AB. 2012.

11. Guyatt G, Oxman AD, Akl EA, Kunz R, Vist G, Brozek J, Norris S, et al. GRADE guidelines: 1. IntroductionGRADE evidence profiles and summary of findings tables. J Clin Epidemiol. 2011;64(4):383-394.

12. Riegler M, Schoppman SF, Bonavina L, Ashton D, Horbach T, Kemen M. Magnetic sphincter augmentation and fundoplication for GERD in clinical practice: one-year results of a multicenter, prospective observational study. Surg Endosc. 2015;29(5):1123-1129.

13. Ganz RA, Edmundowicz SA, Taiganides PA, Lipham JC, Smith CD, DeVault KR, Horgan S, et al. Long-term outcomes of patients receiving a magnetic sphincter augmentation device for gastroesophageal reflux. Clin Gastroenterol Hepatol. 2016;14(5):671-677.

14. Reynolds JL, Zehetner J, Bildzukewicz N, Katkhouda N, Dandekar G, Lipham JC. Magnetic sphincter augmentation with the LINX device for gastroesophageal reflux disease after U.S. Food and Drug Administration approval. Am Surg. 2014;80(10):1034-1038.

15. Schwameis K, Schwameis M, Zorner B, Lenglinger J, Asari R, Riegler FM, Schoppmann SF. Modern GERD treatment: feasibility of minimally invasive esophageal sphincter augmentation. Anticancer Res. 2014;34(5):2341-2348.

16. Smith CD, DeVault KR, Buchanan M. Introduction of mechanical sphincter augmentation for gastroesophageal reflux disease into practice: early clinical outcomes and keys to successful adoption. J Am Coll Surg. 2014;218(4):776-781.

17. Bonavina L, Saino G, Bona D, Sironi A, Lazzari V. One hundred consecutive patients treated with magnetic sphincter augmentation for gastroesophageal reflux disease: 6 years of clinical experience from a single center. J Am Coll Surg. 2013;217(4):577-585.

18. Kappelle WF, Bredenoord AJ, Conchillo JM, Ruurda JP, Bouvy ND, van Berge Henegouwen MI, Chiu PW, et al. Electrical stimulation therapy of the lower oesophageal sphincter for refractory gastro-oesophageal reflux disease - interim results of an international multicentre trial. Aliment Pharmacol Ther. 2015;42(5):614-625.

19. Rodriguez L, Rodriguez P, Gomez B, Ayala JC, Oxenberg D, Perez-Castilla A, Netto MG, et al. Two-year results of intermittent electrical stimulation of the lower esophageal sphincter treatment of gastroesophageal reflux disease. Surgery. 2015;157(3):556-567.

20. Rodriguez L, Rodriguez PA, Gomez B, Netto MG, Crowell MD, Soffer E. Electrical stimulation therapy of the lower esophageal sphincter is successful in treating GERD: long-term 3-year results. Surg Endosc. 2016;30(7):2666-2672.

21. Bonavina L, Saino GI, Bona D, Lipham J, Ganz RA, Dunn D, DeMeester T. Magnetic augmentation of the lower esophageal sphincter: results of a feasibility clinical trial. J Gastrointest Surg. 2008;12(12):2133-2140.

22. Attwood SE. Electrical stimulation for gastroesophageal reflux disease: formal randomized clinical trials are needed. Surgery. 2015;157(3):568-569.

23. Erdos J, Stanak M. Magnetic sphincter augmentation device (MSAD) in patients with gastroesophageal reflux disease (GERD). Decision Support Document No.101, Ludwig Boltzmann Institute for Health Technology Assessment, Vienna. 2016.

24. Skubleny D, Switzer NJ, Dang J, Gill RS, Shi X, de Gara C, Birch DW, et al. LINX((R)) magnetic esophageal sphincter augmentation versus Nissen fundoplication for gastroesophageal reflux disease: a systematic review and meta-analysis. Surg Endosc. 2017;31(8):3078-3084.

25. Chen MY, Huang DY, Wu A, Zhu YB, Zhu HP, Lin LM, Cai XJ. Efficacy of magnetic sphincter augmentation versus nissen fundoplication for gastroesophageal reflux dis- 
ease in short term: a meta-analysis. Can J Gastroenterol Hepatol. 2017;2017:9596342.

26. Tofthagen $\mathrm{C}$. Threats to validity in retrospective studies. J Adv Pract Oncol. 2012;3(3):181-183.

27. Rodriguez L, Rodriguez P, Gomez B, Ayala J, Oxenberg D, Perez-Castilla A, Netto M, et al. Electrical stimulation therapy (EST) of the lower esophageal sphincter (LES) is successful in treating GERD-long-term 3 year results. Surgical Endoscopy and Other Interventional Techniques.
2015;29:S340.

28. Kahrilas PJ. Patient information: Acid reflux (gastroesophageal reflux disease) in adults (beyond the basics). In: Talley NJ (ed) UpToDate, Wolters Kluwer. 2016.

29. Bonavina L, DeMeester T, Fockens P, Dunn D, Saino G, Bona D, Lipham J, et al. Laparoscopic sphincter augmentation device eliminates reflux symptoms and normalizes esophageal acid exposure: one- and 2-year results of a feasibility trial. Ann Surg. 2010;252(5):857-862. 